\title{
Utilization of the Safe Surgical Dislocation Approach in the Surgical Management of Early Hip Tuberculosis
}

\author{
Mahmoud AN*, Mahran MA, Abdelwahab MR, \\ El- Husseini TF, Osman WS, Abdledayem SM, \\ Tharwat SM and Eid MAM \\ Department of Orthopedic Surgery, Ain Shams University \\ Hospital, Egypt \\ *Corresponding author: Ahmed Nageeb Mahmoud. \\ Department of Orthopedic Surgery, Ain Shams University \\ Hospital, Egypt
}

Received: July 03, 2017; Accepted: August 24, 2017;

Published: August 31, 2017

\section{Introduction}

While the Osteo-Articular Tuberculosis (OATB) is relatively of rare occurrence in clinical practice, tuberculous arthritis is associated with high risk of severe deterioration of the involved joints. TB of the hip may result in gross deformities, end stage articular cartilage and bone damage, and marked limitation of the patients' quality of life [1]. Early diagnosis, proper management and careful follow up could preserve the joint function in patients with hip TB.

We present a case of early hip tuberculosis, managed successfully through the safe surgical dislocation approach, as described by Ganz et al. [2]. We believe this surgical approach could be very useful in the management of early stages of hip tuberculosis, as well as in cases of chronic resistant hip sepsis generally, to establish the diagnosis and access a deeply seated septic lesion.

\section{Case Presentation}

A female patient, 24 years old, referred to the orthopedic clinic of our university hospital on March 2013 with severe right hip pain and limitation of weight bearing on the right lower limb with a late use of wheelchair during the last 1 week. She gave a history of open surgical debridement for right hip infection twice during the previous 9 months before presentation, with temporary improvement after each surgery. Medical history was positive for asthmatic bronchitis, controlled on anti-asthma medications.

Positive findings on local examination included right hip anterolateral scar, loss of weight bearing on right lower limb; local tenderness and marked limitation of the right hip range of motion due to pain. The pre-operative Oxford hip score was 52 .

Laboratory investigations included a serum Hemoglobin of 10.8 $\mathrm{gm} / \mathrm{dl}$, ESR 1st hour of 60 and CRP of 27 (normal reference up to 5). Available results of the previous surgical biopsies showed no bacterial growth. A trial for hip needle aspiration revealed a limited amount of serous fluid that was negative for bacterial growth. A routine pre operative viral markers check for hepatitis and HIV were negative.

Chest radiograph showed a non specific accentuation of bronchovascular markings (Figure 1A). Plain radiographs of the pelvis and hips (Figure 1B, C) showed a radiolucent oval shadow, occupying the lower portion of the right acetabulum and obscuring the tear drop. Hip CT scan (Figure 1D, 1E) showed a destructive lesion in the posterior column of the right acetabulum, invading into the hip joint cavity and the posterior extra articular space. A sequestrum was noticed inside the posterior column. Hip MRI (Figure 1F) Showed marked synovial thickening, posterior column hyper echoic lesion and mild joint effusion.

Due to severity of the symptoms and failure of the previous surgical attempt; arthrotomy, wide surgical debridement with subtotal synovectomy, sequestrectomy with obtaining multiple biopsies from the synovium and from inside the posterior column were decided. The surgical hip dislocation approach seemed to be most appropriate for performing all planned surgical procedures.

The safe surgical hip dislocation approach as described by Ganz et al. [2] was performed. Through a lateral decubitus position, the surgical steps included a posterolateral hip approach with preservation of the triceps coxae tendons to protect the deep branch of medial circumflex femoral artery, a sliding trochanteric osteotomy, a Z shaped capsulotomy and surgical anterior hip dislocation. With a non-obscured $360 \mathrm{o}$ view of the femoral head and the acetabulum, marked hip synovial thickening, synovial granulomatous lesions in the acetabulum and on the anterior femoral head (Figure 2A,2B) and mild serous effusion were noticed. After performing subtotal synovectomy and joint debridement, a sinus connecting the intra aricular space to the posterior acetabular column was seen and curetted. Sequestrectomy and curette of the posterior acetabular column were done through the sinus. Multiple biopsies were taken from the deep lesion inside the posterior column and the granulomatous soft tissue, and sent for bacteriological and microscopic examination. After completing the joint debridement and copious joint irrigation, a drill hole into the femoral head with $2 \mathrm{~mm} \mathrm{~K}$ wire was done to ensure intact blood supply. The hip was carefully relocated, the capsule was loosely sutured and the trochanter was fixed with two 6.5 screws (Figure $2 \mathrm{C}, 2 \mathrm{D})$. The fascia lata, subcutaneous tissue and skin were closed with a drain that was removed on next post operative day. Tissue biopsies were sent for histological and bacteriological examinations.

Post operative, passive ROM exercises were started one day after the surgery. After 5 days, the results of culture and sensitivity
Austin J Orthopade \& Rheumatol - Volume 4 Issue 2 - 2017 ISSN: 2472-369X | www.austinpublishinggroup.com Mahmoud et al. (C) All rights are reserved
Citation: Mahmoud AN, Mahran MA, Abdelwahab MR, El- Husseini TF, Osman WS, Abdledayem SM, et al. Utilization of the Safe Surgical Dislocation Approach in the Surgical Management of Early Hip Tuberculosis. Austin J Orthopade \& Rheumatol. 2017; 4(2): 1055. 

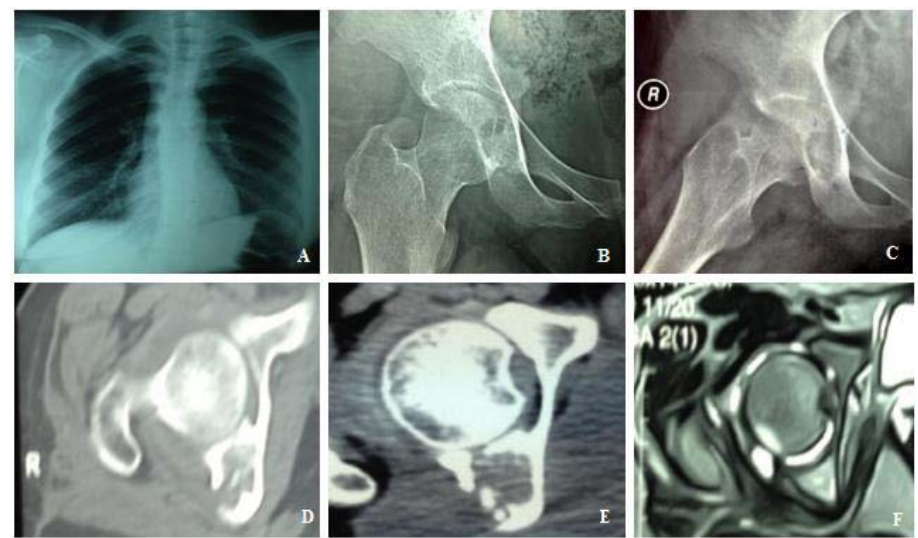

Figure 1: (A) Plain chest X-ray showing non-specific increase of bronchovascular markings. (B, C) Pre operative radiographs showing an oval radiololucent lesion occupying the posterior column. (D, E) Pre operative hip CT scan showing a destructive lesion and sequestrum occupying the posterior acetabular column. (F) Pre operative hip MRI showing synovial thickening, posterior column hyper echoic lesion and mild joint effusion.
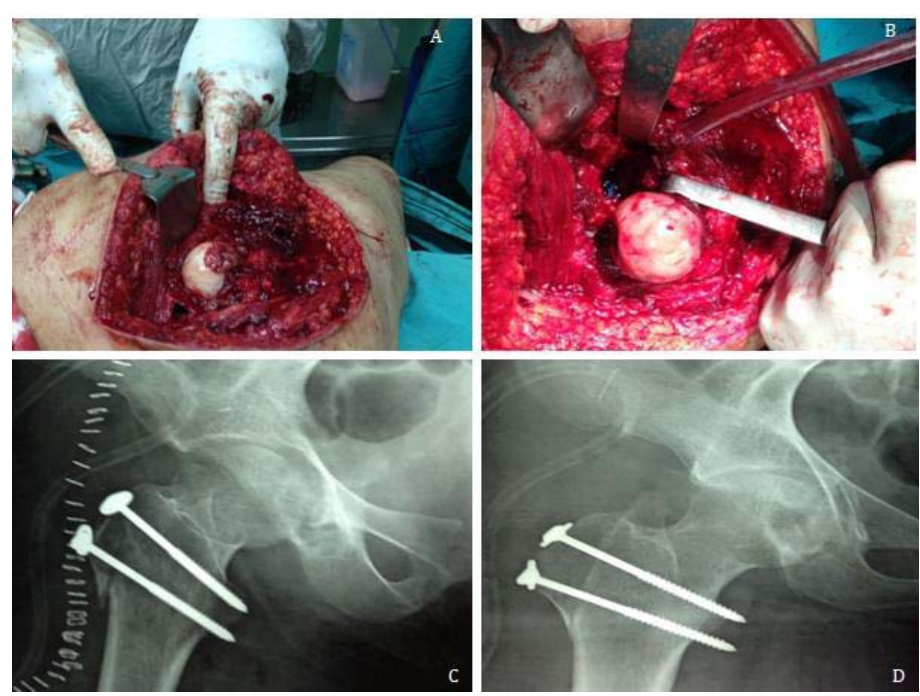

Figure 2: $(A, B)$ Intra operative photograph of the case showing the surgically dislocated femoral head and the acetabular cavity. Note the grayish granulomatous lesion noticed encroaching on the femoral head on dislocation. (C, D) Immediate post operative hip radiograph showing fixation of the trochanteric osteotomy.

showed no bacterial growth, yet the pathology report from the deep sample from the posterior column showed a caseating granuloma of tuberculosis. The histologist tried to stain a specimen with Zeihl Neelsen stain and it was suspicious for the presence of acid fast bacilli. A positive TB DNA with PCR from the tissue specimen was confirmed the diagnosis. The patient was discharged after 10 days on oral anti TB chemotherapy. Toe touch weight bearing was allowed to start after two weeks, and full weight bearing after 6 weeks.

The patient received anti tuberculosis chemotherapy regimen, in the form of Isoniazid (INH) $300 \mathrm{mg} /$ day, Rifampicin $600 \mathrm{mg} /$ day, Ethambutol $15 \mathrm{mg} / \mathrm{kg}$ per day and Pyrazinamide $20 \mathrm{mg} / \mathrm{kg}$ per day for 2 months, then INH and Rifampicin only for another 10 months, as recommended by infectious disease specialist based on the US Center for Disease Control and Prevention (CDC) guidelines [3] for treatment of extra pulmonary TB.

Radiographic examinations were done monthly for following the TB lesion and the trochanteric osteotomy union. Six months after surgery, complete resolution of the patient symptoms was reported and the Oxford hip score was improved to 18. Radiographic examination revealed complete union of the trochanteric osteotomy, with Brooker class 2 heterotropic ossification, and partial healing of the acetabular defect (Figure 3A). The patient stopped the antituberculous medications after the 12 months regimen. Hip MRI was done 12 months post operative and healing of the posterior column lesion was noticed along with preservation of the femoral head shape and vascularity (Figure 3B). Most recent radiographic follow up (50 months post operative) showed healing of the bony lesion (Figure 3C). Until the last clinical follow up, no relapse has occurred and the patient was seen to have a full hip ROM along with pain free full weight bearing.

\section{Discussion}

This report describes a successful management of a case of early hip TB, through the Ganz surgical hip dislocation approach. This approach have helped in confirmation of the diagnosis, and 


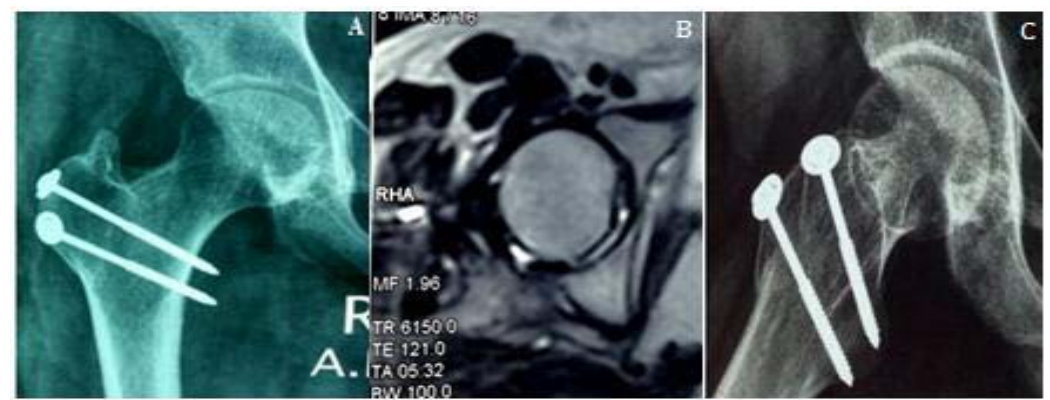

Figure 3: (A) Plain radiographs 6 months post operative showing healing of the bony lesion and trochanteric osteotomy. Note the Brooker class 2 heterotrophic ossification of the healed trochanteric fragment. (B) Hip MRI 12 months post operative showing resolution of the posterior column edema and proves integrity of femoral head. (C) Plain radiographs 52 months post operative showing healing of the lesion and preservation of the joint space.

performing a radical surgical debridement.

The surgical hip dislocation approach has been introduced by Ganz et al. [2], to safely access intra articular hip pathology without risking the femoral head blood supply. It's indications have been increased to include wide spectrum of hip problems, such as femoroacetabular impingement, management of slipped capital femoral epiphysis, Perthes disease sequelae, intra-articular bone tumors, retrieval of foreign bodies from the hip, resurfacing arthroplasty and assisting open reduction and fixation of articular hip fractures [2,4]. To our knowledge, this is the first time to report the use of this approach in management of active hip sepsis.

In previous literature, dislocation of the hip joint during surgical management of hip TB was discouraged, to avoid damage to the femoral head blood vessels and also to avoid iatrogenic fractures in cases with osteoporotic bone [1]. However, these recommendations were not directed to the Ganz technique [2] which should not harm the femoral head blood supply. Also, the relatively easy joint dislocation procedure after performing the trochanteric osteotomy could limit the fracture risk with careful surgical procedure. In cases with deeply seated lesion that is difficult to be accessed through simple arthrotomy or hip arthroscopy, the Ganz technique could present a safe and effective approach for performing wide surgical debridement, total synovectomy and sequestrectomy. Also, it allows for obtaining deep tissue biopsies and establishing the diagnosis in cases of recurrent and resistant hip sepsis, which should call for suspicion against infection with unusual micro organisms, such as TB.

\section{Epidemiology of TB}

Tuberculosis (TB) is an infectious disease caused by Mycobacterium Tuberculosis (M. TB) mainly, and M. Bovis and M. Africanum in rare cases $[1,5]$. The prevalence of TB is evidently high among AIDS patients, and sometimes it is the first manifestation of HIV infection [6]. Latest estimates of the World Health Organization have stated that there were 9.6 million new TB cases in the year 2014 and 1.5 million deaths resulting from $\mathrm{TB}$, with about 1.1 million deaths were among HIV-negative cases and 0.4 million were HIVassociated TB deaths [7].

OATB represents about 1-5\% of all TB cases, half of them occur in the spine. Being the second most affected site, hip TB can account for $15 \%$ of OATB, causing variable presentations that may end with gross deformity and complete destruction of the joint. The major problems that may lead to unfavorable outcomes are the delay in diagnosis and inadequate therapy secondary to patients' non-compliance $[1,3,5]$.

\section{Pathogenesis of hip TB}

Hip TB is usually secondary to hematogenous spread from a primary focus in the lung, lymph nodes or the viscera specially the kidney. The bacteria may lodge either in the synovium first then extends to invade the subchondral bone, or it may lodge first in the bone of head or neck of femur, greater trochanter or acetabulum. In either ways, a destructive process starts in the affected location and progresses to include the all joint. Collection of the necrotic tissue and caseation material may lead to formation of a cold abscess that may perforate the capsule to present around the hip joint in the femoral triangle, medial, lateral or posterior aspects of thigh [1,5,8-10].

\section{Clinical manifestations}

Although any age is susceptible, tuberculous affection of the hip starts usually during first three decades. The patients usually complain of long standing pain in the hip, severe night pain, limp and restriction of movements. Constitutional symptoms of TB as recurrent low grade fever, weight loss, night sweats may be present also. Depending upon the extent of involvement, the clinical signs of the disease may show fullness of the joint, joint stiffness, deformity, shortening of the limb, sinuses and enlarged inguinal lymph nodes [1,5]. Babhulkar and Pand [1] introduced a classification based on the clinic-radiological presentations, into stage of synovitis, early arthritis, arthritis and advanced arthritis.

\section{Diagnosis of the hip TB}

In endemic areas, the clinical manifestations with the radiological signs may be sufficient to diagnose TB and start the chemotherapy. However, in areas where the TB is not common, a high index of suspicion should be taken for diagnosis, especially in immune compromised, HIV patients, or patients with chronic, recurrent joint infection not responding to surgical treatment or associated with negative ordinary bacterial cultures $[1,5,10]$. In all cases, along with the histologic manifestation of TB caseating granuloma, a clear TB diagnosis can be done with detection of the acid fast TB bacilli using Zeihl Neelsen stain in the tissue biopsy or aspirate, which could be positive in only $10-30 \%$ of cases [8]. Detection of TB DNA by PCR in tissue biopsies is also a highly sensitive diagnostic tool. Tissue specimens may be obtained through joint aspirate, open biopsy or with arthroscopic synovial biopsy $[1,5,8]$. 


\section{General principles of Treatment of Tuberculosis}

Due to complex and variable presentations of the hip TB, treatment should be tailored according to the clinical presentation, radiological findings, patient cultural background and activity. In all cases, anti TB chemotherapy should be started once the diagnosis is confirmed. Some literature recommends induction trial of anti TB chemotherapy before clear diagnosis is done in endemic areas $[5,8]$. The optimal duration of treatment of skeletal tuberculosis was mentioned differently in literature, and ranges from 6-24 months $[3,5,10]$. Current recommendations of US CDC [3] for treatment of osseous tuberculosis includes a two-month initial phase of isoniazid, rifampicin, pyrazinamide, and ethambutol followed by 6-12 months regimen of isoniazid and Rifampicin. However, many surgeons prefers to continue osteoarticular TB treatment till there is adequate radiological evidence of healing, which can take up to 18-24 months $[5,10]$. In our case, a total of 12 months regimen was utilized.

\section{Treatment of hip TB according to the Clinico- Radiological Presentation}

Hip pain, limitation of movement with absence of radiological signs may define the early synovitis stages. These patients usually have a very good prognosis after chemotherapy, traction, rest and then mobilization exercises. Failure to respond to the conservative treatment or presence of an established sequestrum or abscess may call for surgical intervention. Synovectomy, sequestrectomy and joint debridement could be done to reduce the diseased tissue load, remove a deep septic focus and establish the diagnosis [1,9-11]. At this stage, the Ganz approach may be helpful in performing such wide surgical debridement, provided that there is good bone quality and no massive joint adhesions or deformity that may hinder the dislocation procedure. With advanced articular surface and osseous destruction or with gross deformities, other treatment modalities like conservative traction regimen to achieve sound ankylosis, open arthrolysis, femoral osteotomies, and excision arthroplasty may be indicated according to disease stage. Hip arthrodesis or total hip arthroplasty are considered as second stage surgery after complete eradication of the septic focus $[1,10,12-14]$.

\section{Conclusion}

The safe surgical dislocation approach could be a good surgical option for management of early cases of hip TB, whenever radical joint debridement, sequestrectomy and synovectomy were planned. It may also be utilized whenever resistant chronic hip infection presents, to establish a diagnosis and perform radical surgical debridement.

\section{References}

1. Babhulkar S, Pande S. Tuberculosis of the hip. Clin Orthop Relat Res. 2002; 398: 93-99.

2. Ganz R, Gill TJ, Gautier E, Ganz K, Krügel N, Berlemann U. Surgical dislocation of the adult hip. A technique with full access to the femoral head and acetabulum without the risk of avascular necrosis. J Bone Joint Surg [Br] 2001; 83: 1119-1124

3. American Thoracic Society, CDC. Infectious Diseases Society of America. Treatment of Tuberculosis. MMWR Recomm Rep. 2003; 52: 1-77.

4. Rebello G, Spencer S, Millis MB, Kim YJ. Surgical dislocation in the management of pediatric and adolescent hip deformity. Clin Orthop Relat Res. 2009; 467: 724-731.

5. Watts HG, Lifeso RM. Tuberculosis of Bones and Joints. J Bone Joint Surg Am. 1996; 78: 288-298.

6. Lupatkin $\mathrm{H}$, Brau N, Flomenberg F, Simberkoff MS. Tuberculous abscesses in patients with AIDS. Clin Infect Dis. 192; 14: 1040-1044.

7. Global tuberculosis report, World Health Organization, 2015. WHO/HTM/ TB/2015.22.

8. Spiegel DA, Singh GK, Banskota AK. Tuberculosis of the Musculoskeletal System. Tech Orthop 2005; 20: 167-178.

9. Tuli SM. General principles of osteoarticular tuberculosis. Clin Orthop Relat Res. 2002; 398: 11-19.

10. Sankaran B. Tuberculosis of bones \& joints. Ind J Tub. 1993; 40: 109-118.

11. Wilkinson MC. Tuberculosis of the hip and knee treated by chemotherapy synovectomy and debridement: A follow-up study. J Bone Joint Surg. 1969; 51: $1343-1359$

12. Katayama R, Itami $Y$, Maruno E. Treatment of hip and knee joint tuberculosis: An attempt to retain motion. J Bone Joint Surg. 1962; 44: 897-917.

13. Kim YY, Han DY, Park BM: Total hip arthroplasty for tuberculous coxarthrosis J Bone Joint Surg. 1987; 69A: 718-727.

14. Johnson R, Barnes KL, Owen R. Reactivation of tuberculosis after total hip replacement. J Bone Joint Surg. 61B: 148-150.
Austin J Orthopade \& Rheumatol - Volume 4 Issue 2 - 2017 ISSN: 2472-369X | www.austinpublishing group.com Mahmoud et al. () All rights are reserved
Citation: Mahmoud AN, Mahran MA, Abdelwahab MR, El- Husseini TF, Osman WS, Abdledayem SM, et al. Utilization of the Safe Surgical Dislocation Approach in the Surgical Management of Early Hip Tuberculosis. Austin J Orthopade \& Rheumatol. 2017; 4(2): 1055 